\title{
Postnatal management of children with antenatal hydronephrosis
}

\author{
Mohammed S. ElSheemy* (1)
}

\begin{abstract}
Background: Postnatal management of infants with antenatal hydronephrosis (ANH) is still one of the most controversial issues. The majority of infants with ANH are asymptomatic with only few children who develop renal insufficiency. Thus, the biggest challenge for pediatric urologists is to distinguish children who will require further investigations and possible intervention prior to the development of symptoms, complications or renal damage in a cost effective manner without exposing them to the hazards of unnecessary investigations.
\end{abstract}

Main body: In this review article, literature on ANH were reviewed to present the current suggestions, recommendations, guidelines and their rational for postnatal management of ANH. It is agreed that a large portion of infants with ANH will improve; thus, the protocol of management is based mainly on observation and follow-up by ultrasound to detect either resolution, stabilization or worsening of hydronephrosis. The first 2 years of life are critical for this followup as the final picture is mostly reached during that period. Advanced imaging using voiding cystourethrography or renal scintigraphy are required for children at risk. Then, surgical intervention is selected only for a subgroup of these infants who showed worsening of hydronephrosis or renal function.

Conclusions: The protocol of management is based mainly on observation and follow-up by US to detect either resolution, stabilization or worsening of hydronephrosis. Postnatal evaluation should be performed for any neonate with a history ANH at any stage during pregnancy even if it was resolved during third trimester. Exclusion of UTI should be performed by urinalysis for all cases followed by urine culture if indicated. Serum creatinine should be performed especially in patients with bilateral ANH. US is the initial standard diagnostic imaging technique. Other imaging modalities like VCUG and nuclear renal scans may be required according to the results of the US evaluation. The most important items in decision making are the presence of bilateral or unilateral hydronephrosis, presence or absence of hydroureter, presence of lower urinary tract obstruction and degree of hydronephrosis on the initial postnatal US. Then an intervention is selected only for a subgroup of these patients who showed deterioration in renal function or degree of hydronephrosis or were complicated by UTIs. All these recommendations are based on the available literature. However, management of ANH is still a controversial issue due to lack of high evidence-based recommendations. Randomised controlled studies are still needed to provide a high level evidence for different aspects of management.

Keywords: Hydronephrosis, Hydroureter, Antenatal, Ultrasound, Voiding cystourethrography, Urinary tract dilation

\section{Background}

Antenatal hydronephrosis (ANH), the dilation of the fetal renal collecting system, is reported in $1-5 \%$ of all pregnancies on prenatal US [1-8]. The widespread use of

${ }^{*}$ Correspondence: mohammedelsheemy@kasralainy.edu.eg

Urology Department, Faculty of Medicine, Cairo University, Cairo, Egypt ultrasonography (US) during pregnancy has resulted in a high detection rate due to the relatively high incidence of congenital anomalies within the urinary tract (UT) in addition to easy detection of the any dilatation or cystic pathology associated with these congenital anomalies [1].

The majority of infants with ANH are entirely asymptomatic at birth. Only few children $(<5 \%$ of neonates
Springer Open

(c) The Author(s) 2020. This article is licensed under a Creative Commons Attribution 4.0 International License, which permits use, sharing, adaptation, distribution and reproduction in any medium or format, as long as you give appropriate credit to the original author(s) and the source, provide a link to the Creative Commons licence, and indicate if changes were made. The images or other third party material in this article are included in the article's Creative Commons licence, unless indicated otherwise in a credit line to the material. If material is not included in the article's Creative Commons licence and your intended use is not permitted by statutory regulation or exceeds the permitted use, you will need to obtain permission directly from the copyright holder. To view a copy of this licence, visit http://creativeco mmons.org/licenses/by/4.0/. 
with $\mathrm{ANH}$ ) develop a renal insufficiency or requires renal transplantation. Thus, the biggest challenge is to distinguish children at risk; who will require further investigations and possible intervention prior to the development of symptoms, complications or renal damage, from those who do not [8-10]. Consequently, some children may be managed by just observation while others may require medical or surgical management. Studying the natural history of ANH thoroughly has affected the protocol of management with shift from a primarily surgical approach to an initial active surveillance with possible intervention for only a selected subgroup of infants who showed or were at risk of complications or deterioration of renal function. Current research tries to detect the different parameters that can classify these children into different risk groups. Unfortunately, there is a lack of prospective randomised studies on ANH. Most of studies were retrospective. Thus, most recommendations are not based on high level of evidence [8-10].

In this review article, literature on $\mathrm{ANH}$ were reviewed to present the current suggestions, recommendations, guidelines and their rational for postnatal management of $\mathrm{ANH}$.

\section{Main text}

A literature search of PubMed from 2000 to 2020 was performed for articles in English reporting on children with prenatal hydronephrosis and who had postnatal evaluation. The types of included articles were practice guideline, clinical Study, clinical Trial, guideline, journal article, meta-analysis, randomized controlled trial, review or systematic review. Different terms were searched including (hydronephrosis or similar terms including oligohydramnios, or ureteral dilation) and (antenatal or near terms including prenatal, newborn, fetal, or natural history). We excluded case reports, editorials, letters, and comments. We excluded also any article in another language than English or performed on adults. Animal studies were excluded. We reviewed 152 articles in depth as they fulfilled our criteria. Reference lists of articles were searched to include any relevant article. This recruited additional 67 articles. The findings and recommendations in these articles were summarized with clarification of similarities or controversies between different studies or guidelines as possible.

\subsection{Etiology}

There is a wide spectrum of conditions that are associated with ANH. These conditions may be pathological or just a transient dilatation. They include transient dilation of the collecting system (41-88\%), upper/lower UT obstructive uropathy including ureteropelvic junction obstruction (UPJ-O) (10-30\%), uretrocele/ectopic ureter
(5-7\%), ureterovesical junction obstruction (UVJ-O) (5\%), posterior urethral valve (PUV) and urethral atresia (1-2\%), non-obstructive processes such as vesicoureteric reflux (VUR) (10-20\%), multicystic dysplastic kidney (MCDK) (4-6\%) and megaureters (5\%). Other rare conditions include prune belly syndrome, cystic kidney disease, congenital ureteric strictures and megalourethra [8].

\subsubsection{Transient hydronephrosis}

It is a transient dilatation which resolves during followup. It may be related to natural kinks and folds that occur during embryological development then disappear with maturation [8]. Mallik et al. [1] conducted a prospective study for 165 infants with ANH to define their natural history. Transient hydronephrosis was detected in $69.69 \%$ of all infants. The rate of transient hydronephrosis was $82.4 \%$ when the cutoff of fetal anteroposterior renal pelvic diameter (APRPD) in the third trimester was determined as $<10 \mathrm{~mm}$ [1]. In another study, the rate of transient hydronephrosis was $40 \%$ in children with ANH of $<12 \mathrm{~mm}$ during the 3rd trimester [11, 12]. Furthermore, it was also reported that surgical intervention was required in only $19-25 \%$ of children with ANH supposed to be due to UPJ-O [13, 14]. Passerotti et al. [15] reported transient hydronephrosis in $52.2 \%$ of the infants (62\% of renal units) that had an antenatal HN [15]. The rate of transient hydronephrosis decreased from $80.6 \%$ in infants with normal first postnatal US to $3.7 \%$ in infants with severe hydronephrosis on first postnatal US [15].

The period of follow-up required for these cases till resolution is not determined as it is variable from case to case. However, the follow-up during the first year is important as most cases of worsening hydronephrosis do that during the first year $[1,8]$.

\subsection{Grading of ANH}

Grading of hydronephrosis is essential for prediction of the risk of potential pathology as well as clinical decision-making. There are many systems used for grading of hydronephrosis. Each grading system is assessed by its objectivity, inter- and intra-observer variations in addition to its ability to describe clearly the renal pathology. The most commonly used grading systems are the APRPD and Society for Fetal Urology (SFU) grading system for hydronephrosis.

\subsubsection{Anteroposterior renal pelvic diameter (APRPD)}

It is the measurement of the APRPD of the renal pelvis as visualized in the transverse plane of the kidney at the hilum. It has a small intra-observer and inter-observer variation [16]. It is the most studied system for assessing ANH in utero $[8,17-19]$. 
The optimal APRPD for diagnosis of ANH is debatable [20]. The incidence of detection of postnatal pathology increases with increasing APRPD that is reported during antenatal evaluation; $11.9 \%, 45.1 \%$ and $88.3 \%$ for mild, moderate and severe hydronephrosis, respectively [21]. Thus, low APRPD cutoffs for diagnosis of ANH will be associated with higher sensitivity in predicting postnatal pathology; however, higher cutoffs will be associated with more specificity [1, 8, 22]. Coplen et al. [23] evaluated retrospectively 257 neonates with ANH and reported that a $15 \mathrm{~mm}$ cut off of APRPD was able to predict $82 \%$ of cases with obstruction which was defined as the need for surgery due to declining function and increasing hydronephrosis. Surgery was performed for UPJ-O (55 cases) and obstructed megaureter (7 cases) [23]. This was confirmed in another study on 53 children in which the best third-trimester fetal APRPD cutoff to predict the need for postnatal surgery was also $15 \mathrm{~mm}$ (sensitivity, 85.7\%; specificity, 94.6\%) [24]. In a prospective study on 213 infants, Ismaili et al. [22] reported that a cut off of $10 \mathrm{~mm}$ in the third trimester predicted only $23 \%$ of renal anomalies after birth. A lower cutoff, $7 \mathrm{~mm}$, was more sensitive with prediction of $68 \%$ of abnormalities [22]. Multiple studies reported that a cutoff of $5 \mathrm{~mm}$ in the second trimester and $7 \mathrm{~mm}$ in the third trimester are the upper limits for normal APRPD and that fetuses presenting with lower APRPD will mostly have no significant pathology in the postnatal evaluation $[4,16,21$, $25-29]$. These cutoffs were reported to be $100 \%$ sensitive in predicting postnatal surgery as reported by Kim et al. [30] while increasing the cutoff to $10 \mathrm{~mm}$ in the third trimester will miss $25 \%$ of UPJ-O cases and $50 \%$ of VUR cases [1]. Maayan-Metzger et al. [28] retrospectively assessed 178 newborn infants with ANH. Of 119 infants with mild ANH (APRPD $<10 \mathrm{~mm}$ ), 116 (97.5\%) showed resolved or mild postnatal hydronephrosis. On the other hand, the rate of detected mild or resolved postnatal hydronephrosis was $80 \%$ (39/49 infants) and $10 \%$ (1/10 infants) for infants with history of moderate or severe ANH, respectively [28]. Kim et al. [30] analyzed APRPD cutoff predicting postnatal surgery in 183 children with ANH (279 renal units); unilaterally in 87 and bilaterally in 96 children. Surgery was performed in 57 children (66 renal units [23.7\%]) after birth. A cutoff of $10 \mathrm{~mm}$ for APRPD during the third trimester had a $97.9 \%$ sensitivity and $23.7 \%$ specificity in predicting postnatal surgery [30]. Longpre et al. [29] tried retrospectively to detect independent predictors for resolution of ANH on 100 children (118 renal units) after excluding cases with VUR, ureterocele, megaureter, or distal ureteric or bladder outlet obstruction. Hydronephrosis resolved spontaneously in $62(52.5 \%)$ units while pyeloplasty was done in 29 (24.5\%) units after a median follow-up was 34 months.
The remaining 27 (22.88\%) units had persistent uncomplicated hydronephrosis at last follow-up. Multivariate analysis showed larger APRPD and SFU grade 4 to be associated with a significantly lower likelihood of resolution. The mean initial APD in resolved cases was $9.4 \mathrm{~mm}$ as opposed to $29 \mathrm{~mm}$ in cases requiring surgery. A cut off of $1.93 \mathrm{~cm}$ or less was associated with resolution, with a positive predictive value of $83 \%$ (sensitivity $100 \%$, specificity $67 \%$ ) [29]. In a meta-analysis performed by Lee et al. [21] on 1308 children from 17 studies, ANH was stratified as mild if APRPD was $\leq 7 \mathrm{~mm}$ in the second trimester and $\leq 9 \mathrm{~mm}$ in the third trimester and severe if $A P R P D \geq 10$ in second trimester and $\geq 15 \mathrm{~mm}$ in the third trimester. The APRPD $>15 \mathrm{~mm}$ in the third trimester predicted an $88 \%$ of postnatal pathology. Taking all of the patients with any degree of $\mathrm{ANH}, 36 \%$ had pathology discovered during postnatal management [21]. Consequently, SFU and Indian Society of Pediatric Nephrology classified ANH as mild if APRPD was $4-6 \mathrm{~mm}$ in the second trimester and 7-9 $\mathrm{mm}$ in the third trimester and severe if APRPD was $>10 \mathrm{~mm}$ and $>15 \mathrm{~mm}$ in the second and third trimester, respectively $[8,9]$. Similarly, neonatal hydronephrosis is diagnosed when APRPD $\geq 7 \mathrm{~mm}$ [9].

The use of APRPD has some limitations which are partly solved by the SFU grading system in which the calyceal dilation and parenchymal changes are taken into consideration $[8,16]$. APRPD is also affected also by the degree of bladder distention, hydration and position of the child [31-37]. Hydration will increase the fluid excretion which will increase both the size of a normal renal pelvis and the bladder volume [32-36]. The APRPD may also decrease when measured in the prone position [38]. Thus, re-evaluation should be performed after bladder emptying. Additionally, follow-up US for each patient should be performed in the same position.

\subsubsection{SFU grading system for hydronephrosis (http://www. uab.edu/images/peduro/SFU/sfu_grading_on_web/ sfu_grading_on_web.htm)}

It is another commonly used grading system which was recommended by SFU for postnatal assessment of hydronephrosis. It integrates multiple measurements including the degree of dilatation of renal pelvis and calyces and the renal parenchyma status [39]. If there is no dilatation of the collecting system, it is grade 0 . If there is a slight splitting of renal sinus (central renal complex, renal pelvis), it is grade 1 . More splitting of the central renal complex with filling of the intrarenal pelvis, filling of the extrarenal pelvis or dilatation of major calyces is graded as 2 . If minor calyces are dilated, it is grade 3 . If this is associated with thinning of the renal parenchyma, it is grade 4 [39]. Thinning of renal parenchyma is defined as a thickness $<50 \%$ of the contralateral side. For bilateral cases, it 
is defined as a thickness $<4 \mathrm{~mm}$. SFU grading system has good intra-rater and moderate inter-rater, reliability [40]. It was reported that the inter-rater reliability and clinical correlation can be improved by sub-classifying SFU grade 4 hydronephrosis into segmental (4A) and diffuse (4B) cortical thinning [41].

\subsubsection{Urinary tract dilation (UTD) classification}

SFU in association with other societies; American College of Radiology, the American Institute of Ultrasound in Medicine, the American Society of Pediatric Nephrology, the Society for Maternal-Fetal Medicine, the Society for Pediatric Urology, the Society for Pediatric Radiology and the Society of Radiologists in Ultrasounds, constructed the UTD classification on 2014, which merges both the APRPD and SFU grading systems trying to abolish their limitations and to unify the description of UT dilation [42]. The consensus panel recommended the use of the term urinary tract dilation instead of other terms including hydronephrosis or pyelectasis [42].

This system can be used for prenatal or postnatal evaluation of hydronephrosis but we will focus here on the postnatal part. It is based on six findings in US. The first point is APRPD which is described as normal if $<10 \mathrm{~mm}$ postnatally. Two findings in UTD classification system are similar to SFU grading which are the parenchymal thickening; described as normal or abnormal, and calyceal dilatation; divided into central [major calyces] and/or peripheral [minor calyces] dilatation. Additionally, there are three other new parameters which are the renal parenchymal appearance; described as normal or abnormal regarding the echogenicity, corticomedullary differentiation and presence of cortical cysts, bladder abnormalities; described as normal or abnormal regarding the wall thickness, presence of ureterocele and presence of dilated posterior urethra, and ureteral condition; described as dilated or not [42]. Based on these findings, UTD classification stratify infants postnatally into three risk categories; UTD P1, P2, and P3. The low risk category (UTD P1) is identified by APRPD 10 to $<15 \mathrm{~mm}$ and/or central calyceal dilation. The intermediate-risk category (UTD P2) is defined as APRPD $\geq 15 \mathrm{~mm}$, peripheral calyceal dilation and/or dilated ureter. Finally, the high-risk category (UTD P3) which is similar to P2 but with parenchymal abnormality (thinning, echogenic and/or decreased corticomedullary differentiation) and/ or bladder abnormality (thickened, ureterocele or posterior urethral dilation) [42]. A multivariate analysis reported that the severity of renal pelvic dilation, ureteral dilation, parenchymal thinning, renal hyperechogenicity, and thickened bladder were independent risk factors for significant pathology in children with ANH. An APRPD $>16 \mathrm{~mm}$; $99.8 \%$ sensitivity and $89.5 \%$ specificity, was predictive for the need for surgery [43]. UTD classification system was reported to be reliable, [44, 45] but was reported to be less reliable than the SFU system in other studies [46]. Hodhod et al. [44] retrospectively compared the reliability of UTD classification system and SFU grading system for postnatal hydronephrosis predicting hydronephrosis resolution and surgical intervention in 490 patients (730 renal units). Hydronephrosis resolved in 357 units (49\%), while a 86 units (12\%) were managed by surgical intervention. The remainder of renal units demonstrated stable or improved hydronephrosis. They reported that UTD system was reliable for evaluation of postnatal hydronephrosis [44]. Multivariate analysis revealed that surgical intervention was predicted by UTD classification system, while SFU grades were predictive of resolution [44]. Rickard et al. [46] compared the intra/inter-rater reliability of SFU and UTD grading systems for hydronephrosis. SFU (1-4) and UTD (1-3) grades were independently assigned by 13 raters (9 pediatric urology staff, 2 nephrologists, 2 radiologists), twice, 3 weeks apart, to 50 sagittal postnatal ultrasonographic views of hydronephrotic kidneys. Overall inter-rater reliability was high for SFU ( $\alpha=0.842$ in session 1 ; and $\alpha=0.808$ in session 2$)$ and moderate for UTD $(\alpha=0.774$ in session 1 and $\alpha=0.679$ in session 2). Overall reliability was significantly higher with the SFU grading system than the UTD classification system. Reliability for intermediate grades (SFU 2/3 and UTD 2) of HN was poor regardless of the system, highlighting the limitations of both classifications in regards to properly segregating moderate HN grades. Intra-rater reliability was considered to be moderate to high with the UTD classification system $(\alpha=0.862$ and 0.723$)$ for sessions 1 and 2 , respectively and high for the SFU grading system for both sessions ( $\alpha=0.923$ for session 1 and $\alpha=0.904$ for session 2). Inter- and intra-rater Reliabilities for SFU and UTD classifications among Urology, Nephrology, and Radiology, as well as between training levels were not significantly different [46].

\subsection{Postnatal evaluation}

\subsubsection{Aim}

Any neonate with ANH should be assessed for the overall health, renal condition, lower UT function, the presence of complications including urinary tract infections (UTIs) in addition to determination of the etiology of ANH. All of these items are important to determine those who will be kept under observation and those who will require intervention [8-10]. The diagnostic strategy should achieve its purpose at a minimal cost and morbidity by avoiding exposure of the infant to unnecessary investigations and interventions but at adequate followup that will preserve the renal parenchyma and prevent 
complications. In most cases, the infants will initially be followed-up regularly then an intervention could be performed for selected patients who showed deterioration in renal function or degree of hydronephrosis or were complicated by UTIs [8-10].

\subsubsection{Indications of postnatal evaluation}

Postnatal evaluation should be performed in any neonate with a history $\mathrm{ANH}$ at any stage during pregnancy even if it was resolved during third trimester [8-10, 27, 47]. This recommendation is stronger if the degree of $\mathrm{ANH}$ was moderate to severe (i.e., fetal pelvic APRPD more than $10 \mathrm{~mm}$ in the 3rd trimester) or increasing in the 3rd trimester compared to the 2nd trimester [1, 21, 47]. This is because the reported incidence of postnatal pathology was higher with the increase in the APRPD that was reported during antenatal evaluation; $11.9 \%, 45.1 \%$ and $88.3 \%$ for mild, moderate and severe hydronephrosis, respectively [21].

\subsubsection{Components}

The diagnostic work-up should involve a detailed history and physical examination including blood pressure measurement [8-10]. The degree of ANH is an important prognostic factor [21]. The presence of a history of oligohydramnios is associated with a poor prognosis and predicts chronic renal failure or even death [48]. Exclusion of UTI should be performed by urinalysis for all cases followed by urine culture if indicated. The urine analysis will discover also the presence of proteinuria [8-10]. Serum creatinine should be performed especially in patients with bilateral ANH [8-10]. For cases with impaired renal function or impaired electrolyte balance (serum bicarbonate, sodium, and potassium), they should be followed twice daily until they plateau [8].

The initial creatinine and blood urea nitrogen (BUN) levels of the newborn may be artificially low due to the effects of maternal renal function mediated through the placenta. Thus, these serum levels should be measured after $48 \mathrm{~h}$ to accurately represent the child's intrinsic renal function [8-10].

Additionally, imaging tests should be performed selectively. US is the initial standard diagnostic technique that should be performed for all infants with history of ANH. However, other imaging modalities like VCUG and nuclear renal scans may be required according to the results of the US evaluation [8-10].

\subsubsection{Timing of US evaluation (Fig. 1)}

US evaluation is better to be delayed to the end of the first week following birth when normal urinary output is established as the physiological neonatal dehydration

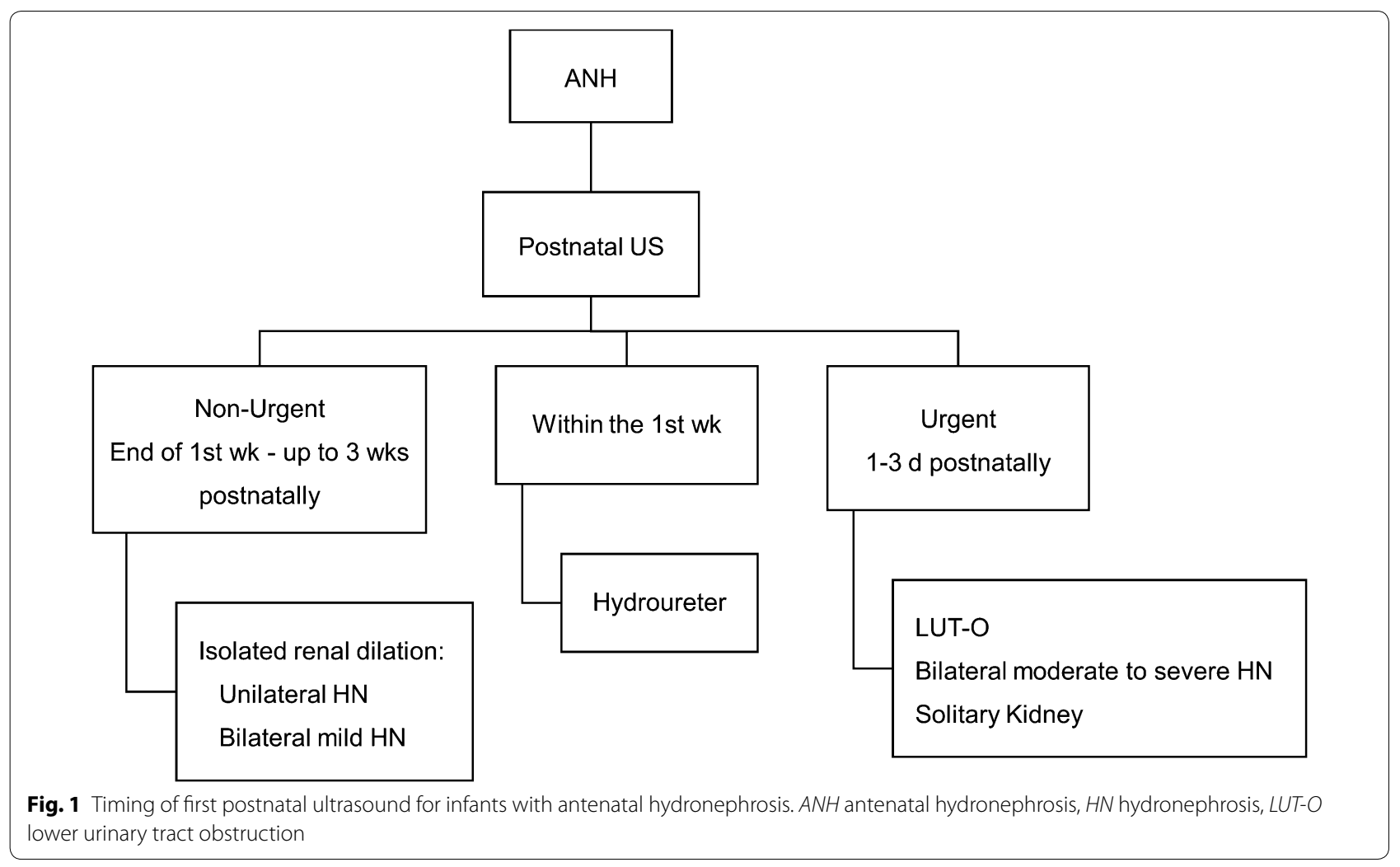


and oliguria lasts $48 \mathrm{~h}$ after birth [1, 8-10, 47, 49-52]. This is accepted for cases with unilateral dilatation in the presence of a normal contralateral kidney. However, an urgent postnatal sonography within 1-3 days is recommended in more severe cases including solitary kidney, bilateral moderate to severe hydronephrosis or when lower UT obstruction is suspected (e.g., oligohydramnios, thick-walled bladder, dilated posterior urethra, urethral obstruction) $[1,8-10,47,49,50,53,54]$. In cases with mild ANH, the postnatal US can be delayed up to 3 weeks following birth [1, 15, 47, 51]. Wiener et al. [50] compared US within $48 \mathrm{~h}$ of birth and at 7 to 10 days of life for infants with $\mathrm{ANH}$ and found an increase in hydronephrosis severity in $44 \%$ of renal units on the second US. An increase of two or greater grades was found in $25 \%$ of renal units. They concluded that the initial US mostly underestimated the degree of hydronephrosis and recommended the initial postnatal sonogram to be performed at 7 to 10 days of life for routine prenatal hydronephrosis but earlier scans may be appropriate in select cases [50].

\subsubsection{What to evaluate in postnatal US?}

All parameters of the different grading systems for $\mathrm{ANH}$; APRPD, SFU grading and UTD classification system, should be evaluated. In order to standardize evaluations, it is recommended to use one of the previously mentioned grading systems $[8-10]$.

\subsection{Further management according to the results of the initial postnatal US evaluation}

These recommendations for management of ANH are derived mainly from the guidelines of the Society for Fetal Urology, the Indian Society of Pediatric Nephrology and Canadian Urological Association [8-10]. Additionally, more recent studies were also included.

The results of the initial postnatal US will help to take the decision of performing a more advanced diagnostic techniques/surgical interventions or just a further followup is required. The most important items in decision making are the presence of bilateral or unilateral hydronephrosis, presence or absence of hydroureter, presence of lower urinary tract obstruction and degree of hydronephrosis on the initial postnatal US.

\subsubsection{Specific situations}

2.4.1.1 Moderate to severe hydronephrosis; bilaterally or in a solitary kidneys, or suspected lower UT obstruction (history of oligohydramnios, thick-walled bladder, dilated posterior urethra) In these cases, prophylactic antibiotics should be given based on prenatal Us and continued until the imaging studies are completed $[1,8,9,53,55]$. The preferred antibiotics include cotrimoxazole (1-2 mg/ $\mathrm{kg} / \mathrm{d})$ or nitrofurantoin $(1 \mathrm{mg} / \mathrm{kg} / \mathrm{d})[9,10,55]$. Amoxicillin or Cephalexin $(10 \mathrm{mg} / \mathrm{kg} / \mathrm{d})$ should replace them in the first 3 months of life $[9,10]$.

Urgent US should be performed within 1-3 days following birth [1, 8-10,53].

VCUG should be performed within 7 days to rule out VUR $[1,8,10,55,56]$. The Indian society for pediatric nephrology recommended to perform VCUG within 3 days for neonates with suspected LUTO due to their higher risk for progressive renal disease and UTI [9]. If VCUG revealed PUV, intervention should be done [8]. If VCUG revealed no reflux, a diuretic renogram is recommended $[8,9]$.

2.4.1.2 Hydroureter The risk of developing UTI is greater in children with dilated ureter than in children with dilation limited to the kidney. Thus, these patients should also be placed on antibiotic prophylaxis based on prenatal Us and continued until imaging studies are complete $[1,8,9,53,55]$.

Postnatal US should be performed within the first 7 days $[1,8,53]$.

Passerotti et al. [15] evaluated infants with ANH and found a higher rate of urinary tract pathology in infants with a ureteric dilation (64.4\% vs $31.8 \%)$ [15]. VCUG is mandatory within 4 weeks $[8,9,44,56]$. If no reflux is found in VCUG, a diuretic renogram is suggested as there is a possibility of VUJ-O $[8,9]$.

Lee et al. assessed non-refluxing hydronephrosis and reported that UTI developed in $47 \%$ of children with hydroureter compared to $13 \%$ without hydroureter and $59 \%$ of children with ureterocele compared to $18 \%$ without ureterocele. They recommended to use continuous antibiotic prophylaxis (CAP) in these infants with hydroureteronephrosis [57]. In another study, Braga et al. [58] reported that hydroureteronephrosis with excluded reflux (primary megaureter) was an independent risk factor for febrile UTI (32\% vs 6\%) compared with isolated hydronephrosis in infants and recommended CAP to be given for these categories [58]. This was confirmed in another study that reported febrile urinary tract infection in $34 \%$ of infants with primary non-refluxing megaureter [59]. Uncircumcised male $(41 \%$ vs $19 \%)$ and lack of continuous antibiotic prophylaxis ( $53 \%$ vs $21 \%$ ) were also independent risk factors for febrile urinary tract infection. Number to be treated (NNT) for the CAP variable was 3 (CAP should be prescribe to 3 children to prevent 1 febrile UTI) while it was 4.5 for circumcision (5 circumcisions would need to be performed to prevent 1 febrile UTI in male infants with primary non-refluxing megaureter) [59]. 


\subsubsection{Isolated renal dilatation (no ureteric dilatation, bladder abnormalities or suspected lower UT obstruction) (Fig. 2)}

2.4.2.1 Severe Hydronephrosis (SFU 4, APD >15 mm) In children with severe hydronephrosis, prophylactic antibiotics should be given based on prenatal Us and continued until the imaging studies are completed $[1,8-10,53,55]$.

VCUG should be performed within 4 weeks to rule out VUR [1, 8-10, 15, 44, 53, 55, 56]. If VCUG revealed reflux, a DMSA scan may be beneficial especially for high grade reflux as it may confirm the presence of renal dysplasia [8]. If reflux is excluded, a diuretic renogram is recommended as there is a high possibility of significant urinary obstruction that will require surgical intervention [8-10, 44, 47]. If VCUG revealed no reflux, antibiotics may be discontinued [8]. However, some authors recommend giving CAP for these children with severe hydronephrosis even after exclusion of reflux $[44,55]$. Lee et al. [57] assessed the relationship between prenatally diagnosed non-refluxing hydronephrosis and UTI in 430 infants. UTI developed in $39 \%$ of children with obstructive uropathy compared to $11 \%$ without obstruction and in $40 \%$ of children with SFU grade 4 hydronephrosis compared to $33 \%$ with grade $3,14 \%$ with grade 2 and $4 \%$ with grade 1 . They recommended to use CAP in these infants with obstructive uropathy or severe hydronephrosis [57]. In another study, Braga et al. [58] reported that high grade hydronephrosis and lack of CAP ( $27 \%$ vs 14\%) emerged as independent risk factors for febrile UTI in infants with ANH. Females (34\% vs $15 \%$ ) and uncircumcised males ( $21 \%$ vs $5 \%$ ) were also risk factors. They recommended CAP to be given for these categories [58]. On the other hand, Easterbrook et al. [60] reported similar UTI rates in infants with ANH regardless of CAP use (9.9\% for CAP vs $7.5 \%$ for no CAP) in their meta-analysis that included 10 non-randomized trials (3909 children). However, subgroup classification according to severity of hydronephrosis could not be performed. Furthermore, there were no available randomized, controlled trials [60]. Thus, continuation of the antibiotic prophylaxis

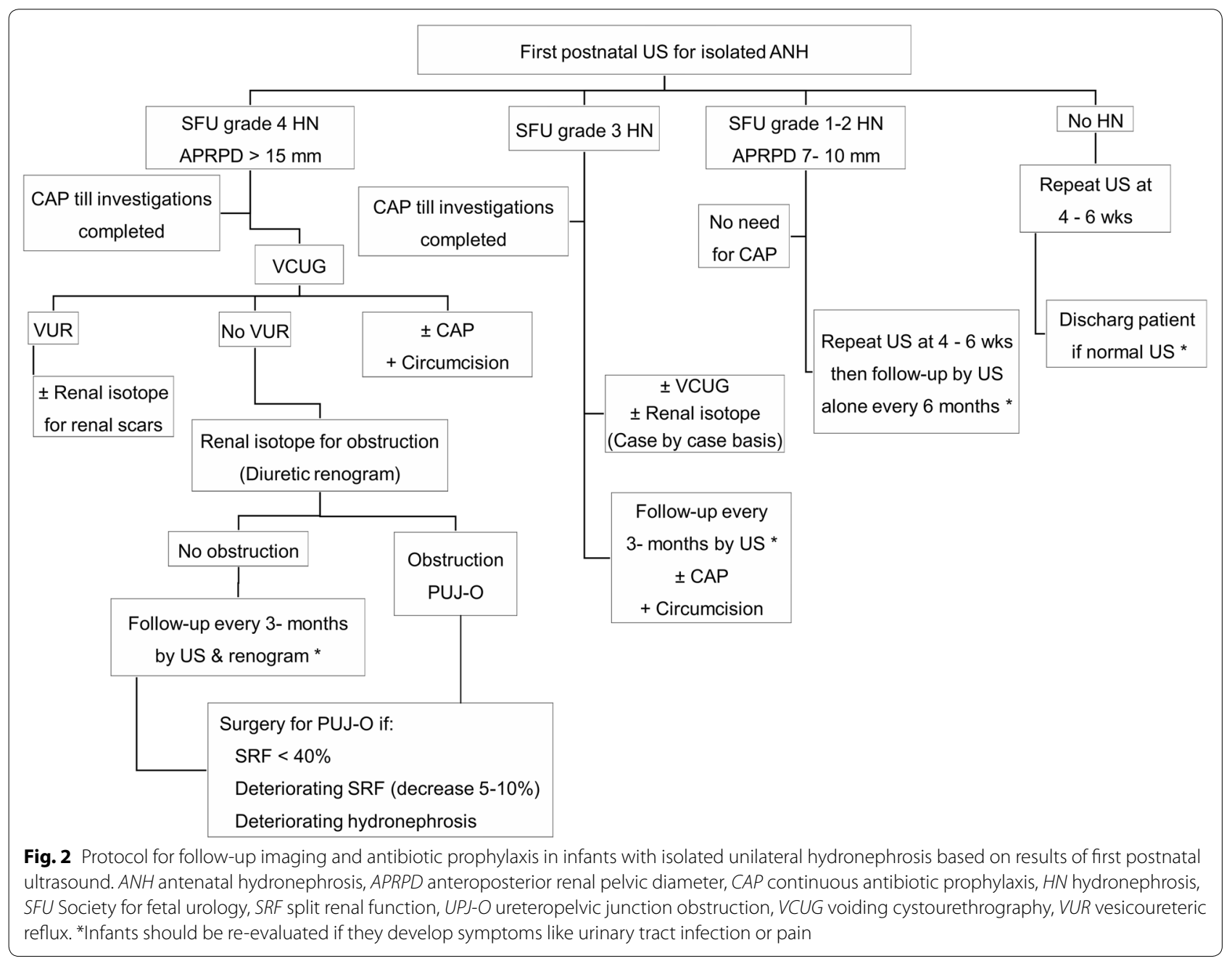


and circumcision for these children is still controversial. However, they should be given for symptomatic cases [8]. The AUA Update Series on ANH suggested CAP for SFU Grade 3 and 4 but stated that it was not evidence-based [61].

2.4.2.2 Moderate Hydronephrosis (SFU 3) Here, the decision is more controversial. The decision should be made on a case by case basis with parental involvement in the decision making. It is equivocal to perform a VCUG or not except in the presence of absolute indications $[8$, $10,21,62]$.

These children should be followed by Us around the age of 3 months. If the hydronephrosis is persistent or increasing especially in the presence of negative VCUG, a baseline MAG3 is useful to exclude obstruction. The MAG3 results will be helpful in determining the timing and role of further studies $[8,52,62]$. However, the Indian society of pediatric nephrology and the Canadian Urological association in addition to other authors recommended VCUG to be performed within 4-6 weeks for these children with SFU grade 3 and renography to be performed for these children if reflux is excluded by VCUG $[1,9,10,15,44,47,52,53]$. If the renogram was also negative with no pathology requiring intervention, both US and renogram can be repeated at the age of 3 months. If the condition is persistent, follow-up should continue to at least 18 months of age [10].

Antibiotic prophylaxis should be taken until the imaging studies are completed, particularly if a VCUG was decided $[8,9,21,53,55,62]$. If VCUG excludes VUR, antibiotic prophylaxis can be discontinued $[8,9,21,62]$.

2.4.2.3 Mild unilateral or bilateral Hydronephrosis (SFU 1-2, APD 7-10 mm) Assessment should be performed by US alone for these children as there is a low incidence of late-occurring progression to significant obstruction $[1,8-10,15,22,23,47,53]$. Additionally, incidence of reflux in these cases is $10-18.9 \%,[15,21,63-65]$ and mostly of low-grade with a high rate of subsequent resolution [1, 54, 63]. Madden-Fuentes et al. [66] reported that isolated SFU grade 1-2 hydronephrosis frequently resolves, with infrequent UTI and minimal progression to surgical intervention in 416 children (623 renal units). Resolution/improvement/stabilization rate was $96.7 \%$ for grade 1 hydronephrosis (66.7\% complete resolution) and $98.7 \%$ for grade 2 hydronephrosis ( $47.6 \%$ complete resolution). One patient with SFU grade 1 and another with SFU grade 2 required surgery. For SFU grades 1-2 hydronephrosis, overall resolution rate was 59.9\% with improvement/stability in $37.6 \%$, and worsening in $2.6 \%$. Only $0.7 \%$ of patients had a febrile UTI. They concluded that antibiotic prophylaxis has a limited role in management of these cases [66]. Similarly, Sencan et al. [67] reported that resolution, improvement, stabilization and worsening rates were $67 \%, 13 \%, 16 \%$, and $3.3 \%$; respectively during follow-up of 760 children with mild hydronephrosis (SFU Grade 1-2) on the first postnatal US [67]. The rate of developed UTI during follow-up was $3.3 \%$. VUR was identified in only $1.7 \%$ out of 475 infants who underwent an initial screening VCUG [67]. Thus, it is recommended to defer VCUG for these cases to avoid its morbidity [1, $10,67]$, as VCUG is invasive with potential risk of UTI in addition to exposure to radiation $[1,68,69]$. Additionally, there is no clear benefit for early diagnosis or treatment of VUR in children with mild hydronephrosis in the absence of lower UT pathology [1, 47, 70, 71].

There is no clear evidence to use antibiotic prophylaxis in these children as the incidence of UTI was very low with no clinical benefit of antibiotic use $[9,10,66,67$, 72-75]. Szymanski et al. [75] evaluated 206 consecutive children with postnatally confirmed prenatally detected hydronephrosis. No UTI was observed in patients with grade 1 hydronephrosis. UTIs in low grade hydronephrosis were only seen in infants who performed VCUG (7 patients) [75]. This low risk of UTI in infants with SFU grade 1-2 was confirmed in a meta-analysis that included 3876 infants from 21 studies. Additionally, it confirmed that there was no benefit of use of prophylactic antibiotics in these children with SFU Grade 1 and 2 or APRPD $<15 \mathrm{~mm}$ as there was no significant difference in the risk of UTI in children with and without antibiotic prophylaxis $(2.2 \%$ vs. $2.8 \%)$. However, there was significant difference in the incidence of UTI favoring use of antibiotic prophylaxis in children with SFU Grade 3 and 4 or $A P R P D \geq 15 \mathrm{~mm}(14.6 \%$ vs. $28.9 \%)$. The estimated number needed to treat to prevent 1 UTI in patients with high-grade hydronephrosis was 7 [76]. Although still debatable, several studies have suggested that circumcision is equally effective alternative to antibiotic prophylaxis in preventing UTI in children with hydronephrosis [42, 62, 76, 77]. The Indian Society of Pediatric Nephrology recommended antibiotic prophylaxis for all infants with documented VUR for the first year of life [9]. This recommendation for use of antibiotic prophylaxis even in low grades of reflux was explained due to the fact that recurrent UTI in children is associated with the risk of new renal scarring in children which increases from $10 \%$ with two UTIs to $60 \%$ after five UTIs $[42,78]$.

\subsubsection{No hydronephrosis (SFU grade $0, A P D<7 \mathrm{~mm}$ )}

A normal initial postnatal US may be detected in $21-32.8 \%$ of children with $\mathrm{ANH} ;[15,27]$ however, $45 \%$ of these children with an initial normal postnatal scan showed an abnormal US at follow-up [27], and 19.4\% diagnosed with pathology during follow-up [15]. In a 
systematic review of 29 studies (1910 infants), the risk of postnatal pathology was $10.8 \%$ in infants with normal or mild hydronephrosis in postnatal US, compared to $69.7 \%$ in infants with moderate-severe hydronephrosis in the postnatal ultrasound [15]. Signorelli reported that out of 51 infants with normalized last antenatal US examination, 8 children (15.6\%) showed hydronephrosis at the 1 month postnatal US examination. Out of these 8 children, one child (12.5\%) required surgical treatment (vesico-ureteral reimplantation) for VUR [79]. Furthermore, it was reported that surgical intervention was required in $5 \%$ of children with a normal US at 1 week of age as it was followed by abnormal US at 1 month of age highlighting the importance of a second check-up at 1 month of life [79]. Clautice-Engle et al. [51] reported that renal obstruction may be underestimated or missed on US obtained 6 days after birth. They found that US obtained 6 weeks after birth is more specific for detecting obstruction in their study on 100 infants (130 kidneys) with ANH. For the 27 kidneys that were obstructed, the mean pelvic diameter increased from $18 \mathrm{~mm}$ (range $5-54 \mathrm{~mm}$ ) on US obtained at 6 days to $22 \mathrm{~mm}$ (range $11-60 \mathrm{~mm}$ ) on US obtained at 6 weeks [51]. Thus, it is recommended to perform a second US at the age of 4-6 weeks if the first postnatal US was normal [8-10, 42, $51,52,55]$. US at that time is more accurate for detection of hydronephrosis $[8-10,42,51]$. If these 2 successive Us within the first 4-6 weeks are normal, significant obstruction is excluded. Additionally, VUR is rare. If VUR is present under these circumstances, it will be of low grade $[9,22,55]$, with a rate of resolution as twice as children with abnormal findings in postnatal US [80]. Furthermore, a normal postnatal US was reported to have a negative predictive value for UTI of $98.9 \%$ [81]. Lidefelt et al. [55] evaluated 103 infants with ANH by performing two US examinations; the first after 5-7 days and the second after 3 weeks of life. Antibacterial prophylaxis was given to those with APRPD $\geq 15 \mathrm{~mm}$, VUR grades III-V and suspected obstruction. In 53/103 babies, both US examinations were normal. Of these 53 infants, 3 (5.6\%) had VUR grade I. During follow-up, 2 (3.7\%) girls developed UTI at 18 and 24 months of age, respectively; both had a normal VCUG. Among the 50 infants with abnormal initial US, $6(12 \%)$ had VUR, of which four were high grade (IV-V). All 4 (8\%) infants with high grade (IV-V) VUR developed UTI. The rate of UTI in these 50 infants was $14 \%$. They reported that UTI is uncommon in infants with two normal postnatal US examinations and recommended not to use antibacterial prophylaxis in these infants [55]. Similarly, it was reported that the risk of VUR on VCUG was $<6.7 \%$ in 74 infants with two normal postnatal ultrasounds (at day 5 and 1 month after birth) compared to $40 \%$ in children with detected hydronephrosis in the postnatal US. However, complications of VCUG occurred in 3.4\% patients despite antibiotic prophylaxis before doing VCUG [68].

\subsection{Subsequent follow-up}

Long-term follow-up for children with ANH is required. However, there is a controversy on the frequency and appropriate length of follow-up. Late worsening or recurrence of hydronephrosis can occur in the first two years of life and occasionally later up to $5-6$ years $[82,83]$. Matsui et al. [82] evaluated 344 children (483 kidneys) with ANH for a mean follow-up of 10 years. Only children with isolated hydronephrosis were included. Pyeloplasty was required in 87 children ( 89 kidneys). Of the remaining 257 children (394 kidneys), only 4 kidneys (1\%) showed initial improvement then worsened later in life. Mean age at worsening of hydronephrosis was 40 months (range 22 to 60 ). All of them presented with clinical symptoms and treated by pyeloplasty [82]. Coplen et al. [23] reported that half of the surgeries required for children with $\mathrm{ANH}$ were performed before the age of 6 months. Only $3 / 62$ children underwent surgery after age 18 months. Of these patients one had increasing hydronephrosis at age 2.5 years and two had symptomatic renal colic at age 6 years. Thus, they reported that progression of hydronephrosis and/or a decrease in renal function most commonly occurs in the first 12 months of life [23]. Similarly, Longpre et al. [29] reported that 29 pyeloplasties were performed at a median age of 19 months [29]. Ulman et al. followed non-operatively 104 infants with isolated severe (SFU grade 3-4) hydronephrosis after exclusion of cases with VUR. Pyeloplasty was required in only $22 \%$ of infants. All of them were younger than 15 months at the time of surgery. The remaining infants had an improvement or resolution of their hydronephrosis. Mean time to maximum improvement or resolution was 30 and 19 months, respectively [84]. Lee et al. [57] reported that UTI developed in $19 \%$ of infants with non-refluxing hydronephrosis. UTI developed before the age 6 months in $84 \%$ of these infants [57]. In another study, Braga et al. [58] reported that febrile UTI developed in $19 \%$ of infants with ANH at a median of 4 months [58]. In another study, the median time to develop febrile UTI was 3.3 months in infants with primary non-refluxing megaureter. Overall $76 \%$ of megaureters resolved during a median followup of 19 months [59].

It is generally agreed that those with moderate and severe hydronephrosis (SFU Grade 3 and 4) require earlier and more frequent postnatal US evaluation than those with mild (SFU Grade 1 and 2) UT dilation [1, 8, $23,42,52,55,83,84]$. Consequently, it is recommended to schedule follow-up visits; at 3-6 months then 6-12 monthly, until resolution for children with detected 
hydronephrosis on the 1-month US with assessment of these children using Us and UTI surveillance at each visit. The follow-up should be less frequent for lower grades (SFU 1-2) [8, 9, 22, 27, 30, 55, 83, 85, 86]. VCUG and/ or renogram will be required for these children if they are complicated during follow-up by UTI, deterioration of hydronephrosis, or parenchymal progressive thinning $[9,23,44,83,87]$. On the other hand, it is recommended by some authors that children with mild hydronephrosis (1-2) on the 1-month US can be discharged from further surveillance $[1,52,53]$, but they should be re-evaluated if they develop UTI or pain [52, 53, 82]. In these circumstances, VCUG and even renogram should be considered $[1,9]$.

\subsection{Precautions and recommendations during conduction of imaging techniques \\ 2.6.1 Voiding cystourethrogram}

There are absolute indications in which VCUG is recommended for children with ANH. This need for VCUG may be urgent within 1 week following birth or less urgent within 1 month. The urgent indications include the suspected cases of infra-vesical obstruction including (PUV), the presence of bilateral moderate to high-grade hydronephrosis or the presence of ureteric dilatation during US examination [8-10]. The less urgent indications include the detection of a duplex kidney or a ureterocele during US examination due to the high incidence of lower pole reflux in these cases. Other less urgent indications include the presence of thick-walled bladder, bladder diverticula, suspected Neurogenic bladder, cortical thinning or increased echogenicity [54]. VCUG is recommended also for children with ANH who developed UTI during follow-up [9]. The VCUG will help to determine the degree of reflux based on which the further management of reflux will be decided. There is a standard classification for VUR provided by the International Reflux Study Committee in 1985 [88]. If the contrast reaches only the ureter, it is grade 1 . If reaching the pelvicalyceal system, it is grade 2 . If there is a dilatation of the pelvicalyceal system, it is grade 3 . If blunting of fornices occurs (loss of angles of fornices), it is grade 4 which is usually associated with moderate dilatation of pelvicalyceal system. If papillary impressions are no longer visible, it is grade 5 which is usually associated with marked dilatation of the collecting system and ureter [88]. In isolated hydronephrosis, there is a controversy on the necessity for VCUG in SFU grade $3[8,10,21,62]$. There are also emerging studies that prefer to start with radionuclide studies for isolated unilateral asymptomatic SFU grade 4 hydronephrosis in the absence of ureteric dilatation to diagnose PUJ-O and to help the surgical decision. If obstruction was confirmed in these cases, VCUG will not affect the treatment decision, operative outcome or postoperative complications as the incidence of associated VUR is very low and mostly of low grade [89].

\subsubsection{Renal scintigraphy}

A radionuclide study can estimate differential renal function, detect renal scarring, which can result from pyelonephritis or renal dysplasia; and determine the severity of obstruction by analysis of the drainage of the kidney. Based on this invaluable data regarding the diagnosis and follow-up of children with ANH data, a decision can be taken for intervention or follow-up [8, 10, 47, 90].

Radionuclide study can used for diagnosis and followup of suspected UPJ-O in children with moderate to severe hydronephrosis with non-dilated ureter and no reflux on VCUG [1, 8-10]. A decision of pyeloplasty can be taken based on a renogram findings when impaired renal function (differential renal function $<40 \%$ ) with impaired drainage $\left(\mathrm{T}^{1} 12>20 \mathrm{~min}\right)$ are detected. Another indication for pyeloplasty is to detect a worsening renal function ( $\geq 5-10 \%$ decline) during follow-up renogram [8-10, 44, 53, 54, 82, 84, 91-93]. Development of symptoms like pain and vomiting or complicating UTI are also indications for surgery [44, 53]. Radionuclide study is also useful for diagnosis of other causes of urinary tract obstruction like suspected UVJ-O as in infants with moderate to severe hydronephrosis associated with dilated ureter and no reflux on VCUG $[1,8,9]$. Similarly, it is also useful for postoperative follow-up of pyeloplasty or ureterovesical implantation for these cases $[8,91]$. It can also differentiate UPJ-O from multicystic dysplastic kidney by confirming total absence of function in children with multicystic kidney [1]. Radionuclide study can also assess the effect on renal scarring and renal function in some cases with VUR which is important for surgical decision [1]. Radionuclide cystogram is also useful for surveillance of VUR or diagnosis of VUR in siblings. It has a lower degree of radiation exposure compared to VCUG [10, 94].

2.6.2.1 Precautions It is better not to be performed before the age of 6-12 weeks to allow for renal maturation. Otherwise, the results will be suboptimal $[8,9,47]$.

A good drainage on renography excludes obstruction. However, poor drainage curve on diuretic renography cannot be relied upon in young infants as it may only reflect delayed emptying of a dilated system but not an obstruction [13, 29, 47, 54, 84, 91, 92, 95]. A large dilated pelvis is not expected to drain as quickly as a smaller volume pelvis especially in infants. This was confirmed in many studies on ANH in which impaired drainage curves were reported in some children but dilatation and differential function remained stable or improved over time, 
with no need for intervention. This is because drainage curve is affected by many factors including degree of dilatation, the degree of maturation of the kidney, the degree of hydration, individual kidney function, urine flow rate, the timing of administration of the diuretic and the timing of post-micturition images. The drainage can also be inhibited by the supine position or the presence of a full bladder [13, 47, 54, 84, 95]. Koff et al. [94] reported that $>50 \%$ of infants with improvement of their hydronephrosis having obstructed T1/2 values. Thus, it was recommended by some authors to avoid using T1/2 in deciding the need for intervention [13, 23, 54, 84, 91, 95]. The SFU and the Pediatric Nuclear Medicine Council of the Society for Nuclear Medicine published guidelines; to standardize all aspects of doing diuretic renogram which were revised again on 2008 [96, 97].

\section{Conclusion}

According to the current literature, it is agreed that a large portion of infants with ANH will improve; thus, the protocol of management is based mainly on observation and follow-up by US to detect either resolution, stabilization or worsening of hydronephrosis. The first 2 years of life are critical for this follow-up as the final picture is mostly reached during that period.

Postnatal evaluation should be performed in any neonate with a history ANH at any stage during pregnancy even if it was resolved during third trimester. Exclusion of UTI should be performed by urinalysis for all cases followed by urine culture if indicated. Serum creatinine should be performed especially in patients with bilateral $\mathrm{ANH}$. US is the initial standard diagnostic imaging technique. US evaluation is better to be delayed to the end of the first week following birth when normal urinary output is established as the physiological neonatal dehydration and oliguria lasts $48 \mathrm{~h}$ after birth. This is accepted for cases with unilateral dilatation in the presence of a normal contralateral kidney. However, an urgent postnatal sonography within 1-3 days is recommended in more severe cases including solitary kidney, bilateral moderate to severe hydronephrosis or when lower UT obstruction is suspected (e.g., oligohydramnios, thick-walled bladder, dilated posterior urethra, urethral obstruction). In cases with mild ANH, the postnatal US can be delayed up to 3 weeks following birth. Other imaging modalities like VCUG and nuclear renal scans may be required according to the results of the US evaluation. The most important items in decision making are the presence of bilateral or unilateral hydronephrosis, presence or absence of hydroureter, presence of lower urinary tract obstruction and degree of hydronephrosis on the initial postnatal US. Then an intervention is selected only for a subgroup of these patients who showed deterioration in renal function or degree of hydronephrosis or were complicated by UTIs.

All these recommendations are based on the available literature. However, management of ANH is still a controversial issue due to lack of high evidence-based recommendations. Randomised controlled studies are still needed to provide a high level evidence for different aspects of management.

\section{Abbreviations \\ ANH: Antenatal hydronephrosis; APRPD: Anteroposterior renal pelvic diameter; CAP: Continuous antibiotic prophylaxis; HN: Hydronephrosis; LUT-O: Lower urinary tract obstruction; MCDK: Multicystic dysplastic kidney; PUV: Posterior urethral valve; SFU: Society for fetal urology; SRF: Split renal function; UPJ-O: Ureteropelvic junction obstruction; UT: Urinary tract; UTD: Urinary tract dila- tion; UTI: Urinary tract infection; UVJ-O: Ureterovesical junction obstruction; VCUG: Voiding cystourethrography; VUR: Vesicoureteric reflux.}

\section{Acknowledgements}

Not applicable.

Authors' contributions

MSE: Performed all aspects of the manuscript. The author read and approved the final manuscript.

\section{Funding}

This research did not receive any specific grant from funding agencies in the public, commercial, or not-for-profit sectors.

\section{Availability of data and materials}

Not applicable (review study).

Ethics approval and consent to participate

Not applicable (review study).

\section{Consent for publication}

Not applicable (review study).

\section{Competing interests}

The authors declare that they have no conflict of interest.

Received: 22 July 2020 Accepted: 17 November 2020

Published online: 07 December 2020

\section{References}

1. Mallik M, Watson AR (2008) Antenatally detected urinary tract abnormalities: more detection but less action. Pediatr Nephrol 23:897-904

2. Ek S, Lidefeldt KJ, Varricio L (2007) Fetal hydronephrosis; prevalence, natural history and postnatal consequences in an unselected population. Acta Obstet Gynecol Scand 86:1463-1466

3. Livera LN, Brookfield DS, Egginton JA, Hawnaur JM (1989) Antenatal ultrasonography to detect fetal renal abnormalities: a prospective screening programme. BMJ 298:1421-1423

4. Sairam S, Al-Habib A, Sasson S, Thilaganathan B (2001) Natural history of fetal hydronephrosis diagnosed on mid-trimester ultrasound. Ultrasound Obstet Gynecol 17:191-196

5. Blyth B, Snyder HM, Duckett JW (1993) Antenatal diagnosis and subsequent management of hydronephrosis. J Urol 149:693-698

6. Gunn TR, Mora JD, Pease P (1995) Antenatal diagnosis of urinary tract abnormalities by ultrasonography after 28 weeks' gestation: incidence and outcome. Am J Obstet Gynecol 172:479-486

7. Persutte WH, Koyle M, Lenke RR, Klas J, Ryan C, Hobbins JC (1997) Mild pyelectasis ascertained with prenatal ultrasonography is pediatrically significant. Ultrasound Obstet Gynecol 10:12-18 
8. Nguyen HT, Herndon CD, Cooper C, Gatti J, Kirsch A, Kokorowski P et al (2010) The Society for Fetal Urology consensus statement on the evaluation and management of antenatal hydronephrosis. J Pediatr Urol 6:212-231

9. Sinha A, Bagga A, Krishna A, Bajpai M, Srinivas M, Uppal R, Agarwal I, Indian Society of Pediatric Nephrology (2013) Revised guidelines on management of antenatal hydronephrosis. Indian Pediatr 50(2):215-231

10. Capolicchio JP, Braga LH, Szymanski KM (2018) Canadian Urological Association/Pediatric Urologists of Canada guideline on the investigation and management of antenatally detected hydronephrosis. Can Urol Assoc J 12(4):85-92

11. Harding LJ, Malone PS, Wellesley DG (1999) Antenatal minimal hydronephrosis: is its follow-up an unnecessary cause of concern? Prenat Diagn 19:701

12. Ransley PG, Dhillon HK, Gordon I, Duffy PG, Dillon MJ, Barratt TM (1990) The postnatal management of hydronephrosis diagnosed by prenatal ultrasound. J Urol 144:584

13. Dhillon HK (1998) Prenatally diagnosed hydronephrosis: the Great Ormond Street experience. Br J Urol 81(Suppl. 2):39

14. Palmer LS, Maizels M, Cartwright PC, Fernbach SK, Conway JJ (1998) Surgery versus observation for managing obstructive grade 3 to 4 unilateral hydronephrosis: a report from the Society for Fetal Urology. J Urol 159:222

15. Passerotti CC, Kalish LA, Chow J, Passerotti AM, Recabal P, Cendron M et al (2011) The predictive value of the first postnatal ultrasound in children with antenatal hydronephrosis. J Pediatr Urol 7:128-136

16. Pereira AK, Reis ZS, Bouzada MC, de Oliveira EA, Osanan G, Cabral AC (2011) Antenatal ultrasonographic anteroposterior renal pelvis diameter measurement: is it a reliable way of defining fetal hydronephrosis? Obstet Gynecol Int 2011:861865

17. Kitchens DM, Herndon CDA (2009) Antenatal hydronephrosis. Curr Uro Rep 10:126

18. Arger PH, Coleman BG, Mintz MC, Snyder HP, Camardese T, Arenson RL et al (1985) Routine fetal genitourinary tract screening. Radiology 156:485

19. Grignon A, Filion R, Filiatrault D, Robitaille P, Homsy Y, Boutin H et al (1986) Urinary tract dilatation in utero: classification and clinical applications. Radiology 160:645-647

20. Ismaili K, Avni FE, Piepsz A, Wissing KM, Cochat P, Aubert D, Hall M (2004) Current management of infants with fetal renal pelvis dilation: a survey by French speaking pediatric nephrologists and urologists. Pediatr Nephrol 19(9):966-971

21. Lee RS, Cendron M, Kinnamon DD, Nguyen HT (2006) Antenatal hydronephrosis as a predictor of postnatal outcome: a meta-analysis. Pediatrics 118(2):586-593

22. Ismaili K, Hall M, Donner C, Thomas D, Vermeylen D, Avni FE, Brussels Free University Perinatal Nephrology Study Group (2003) Results of systematic screening for minor degrees of fetal renal pelvis dilatation in an unselected population. Am J Obstet Gynecol 188:242-246

23. Coplen DE, Austin PF, Yan Y, Blanco VM, Dicke JM (2006) The magnitude of fetal renal pelvic dilatation can identify obstructive postnatal hydronephrosis, and direct postnatal evaluation and management. J Urol 176(2):724-727

24. Shamshirsaz AA, Ravangard SF, Egan JF, Prabulos AM, Shamshirsaz AA, Ferrer FA et al (2012) Fetal hydronephrosis as a predictor of neonatal urologic outcomes. J Ultrasound Med 31(6):947-954

25. Chitty LS, Altman DG (2003) Charts of fetal size: kidney and renal pelvis measurements. Prenat Diagn 23:891-897

26. Scott JE, Renwick M (2001) Antenatal renal pelvic measurements: what do they mean? Br J Urol Int 87:376-380

27. Aksu N, Yavapcan O, Kangin M, Kara OD, Aydin Y, Erdoðan H et al (2005) Postnatal management of infants with antenatally detected hydronephrosis. Pediatr Nephrol 20:1253-1259

28. Maayan-Metzger A, Lotan D, Jacobson JM, Raviv-Zilka L, Ben-Shlush A Kuint J, Mor Y (2011) The yield of early postnatal ultrasound scans in neonates with documented antenatal hydronephrosis. Am J Perinatol 28(8):613-618

29. Longpre M, Nguan A, Macneily AE, Afshar K (2012) Prediction of the outcome of antenatally diagnosed hydronephrosis: a multivariable analysis. J Pediatr Urol 8(2):135-139. https://doi.org/10.1016/j.jpurol.2011.05.013

30. Kim HJ, Jung HJ, Lee HY, Lee YS, Im YJ, Hong CH et al (2012) Diagnostic value of anteroposterior diameter of fetal renal pelvis during second and third trimesters in predicting postnatal surgery among Korean population: useful information for antenatal counseling. Urology 79(5):1132-1137

31. Leung VY, Chu WC, Metreweli C (2009) Hydronephrosis index: a better physiological reference in antenatal ultrasound for assessment of feta hydronephrosis. J Pediatr 154(1):116-120

32. Angell SK, Pruthi RS, Shortliffe LD (1998) The urodynamic relationship of renal pelvic and bladder pressures, and urinary flow rate in rats with congenital vesicoureteral reflux. J Urol 160(1):150-156

33. Evans ED, Meyer JS, Harty MP, Bellah RD (1999) Assessment of increase in renal pelvic size on post-void sonography as a predictor of vesicoureteral reflux. Pediatr Radiol 29:291-294

34. Hvarness H, Jakobsen H, Hermansen F, Marving J, Meyhoff HH (1999) Effect of a full bladder on urine production in humans. Scand J Urol Nephrol 33:386-391

35. Jones DA, Lupton EW, George NJ (1990) Effect of bladder filling on upper tract urodynamics in man. Br J Urol 65:492-496

36. Peerboccus M, Damry N, Pather S, Devriendt A, Avni F (2013) The impact of hydration on renal measurements and on cortical echogenicity in children. Pediatr Radiol 43:1557-1565

37. Carrico CW, Zerin JM (1996) Sonographic measurement of renal length in children: does the position of the patient matter? Pediatr Radiol 26:553-555

38. Sharma G, Sharma A, Maheshwari P (2012) Predictive value of decreased renal pelvis anteroposterior diameter in prone position for prenatally detected hydronephrosis. J Urol 187:1839-1843

39. Fernbach SK, Maizels M, Conway JJ (1993) Ultrasound grading of hydronephrosis: introduction to the system used by the Society for Fetal Urology. Pediatr Radiol 23(6):478-480

40. Keays MA, Guerra LA, Mihill J, Raju G, Al-Asheeri N, Geier P et al (2008) Reliability assessment of Society for Fetal Urology ultrasound grading system for hydronephrosis. J Urol 180(4 Suppl):1680-1682 (discussion 1682-3)

41. Sibai H, Salle JL, Houle AM, Lambert R (2001) Hydronephrosis with diffuse or segmental cortical thinning: impact on renal function. J Urol 165(6 Pt 2):2293-2295

42. Nguyen HT, Benson CB, Bromley B, Campbell JB, Chow J, Coleman B et al (2014) Multidisciplinary consensus on the classification of prenatal and postnatal urinary tract dilation (UTD classification system). J Pediatr Urol 10(6):982-998

43. Dias CS, Silva JM, Pereira AK, Marino VS, Silva LA, Coelho AM et al (2013) Diagnostic accuracy of renal pelvic dilatation for detecting surgically managed ureteropelvic junction obstruction. J Urol 190:661-666

44. Hodhod A, Capolicchio JP, Jednak R, El-Sherif E, Ael-A El-Doray, ElSherbiny M (2016) Evaluation of urinary tract dilation classification system for grading postnatal hydronephrosis. J Urol 195(3):725-730

45. Chalmers DJ, Meyers ML, Brodie KE, Palmer C, Campbell JB (2016) Inter-rater reliability of the APD, SFU, and UTD grading systems in fetal sonography and MRI. J Pediatr Urol 12(5):305.e1-305.e5

46. Rickard M, Easterbrook B, Kim S, Farrokhyar F, Stein N, Arora S et al (2017) Six of one, half a dozen of the other: a measure of multidisciplinary inter/ intra-rater reliability of the society for fetal urology and urinary tract dilation grading systems for hydronephrosis. J Pediatr Urol 13(1):80.e1-80.e5

47. Vemulakonda V, Yiee J, Wilcox DT (2014) Prenatal hydronephrosis: postnatal evaluation and management. Curr Urol Rep 15(8):430 (Review)

48. Oliveira EA, Diniz JS, Cabral AC, Leite HV, Colosimo EA, Oliveira RB et al (1999) Prognostic factors in fetal hydronephrosis: a multivariate analysis. Pediatr Nephrol 13(9):859-864

49. Dejter SW Jr, Gibbons MD (1989) The fate of infant kidneys with fetal hydronephrosis but initially normal postnatal sonography. J Urol 142:661-662

50. Wiener JS, O'Hara SM (2002) Optimal timing of initial postnatal ultrasonography in newborns with prenatal hydronephrosis. J Urol 168:1826-1829

51. Clautice-Engle T, Anderson NG, Allan RB, Abbott GD (1995) Diagnosis of obstructive hydronephrosis in infants: comparison sonograms performed 6 days and 6 weeks after birth. AJR Am J Roentgenol 164:963-967

52. Riccabona M, Avni FE, Blickman JG, Dacher J-N, Darge K, Lobo ML et al (2008) Imaging recommendations in paediatric uroradiology: minutes of the ESPR workgroup session on urinary tract infection, fetal hydronephrosis, urinary tract ultrasonography and voiding 
cystourethrography, Barcelona, Spain, June 2007. Pediatr Radiol 38:138-145

53. Wollenberg A, Neuhaus TJ, Willi UV, Wisser J (2005) Outcome of feta renal pelvic dilatation diagnosed during the third trimester. Ultrasound Obstet Gynecol 25(5):483-488

54. Belarmino JM, Kogan BA (2006) Management of neonatal hydronephrosis. Early Hum Dev 82:9-14

55. Lidefelt KJ, Herthelius M (2008) Antenatal hydronephrosis: infants with minor postnatal dilatation do not need prophylaxis. Pediatr Nephrol 23(11):2021-2024

56. Skoog SJ, Peters CA, Arant BS Jr, Copp HL, Elder JS, Hudson RG et al (2010) Pediatric Vesicoureteral Reflux Guidelines Panel summary report: clinical practice guidelines for screening siblings of children with vesicoureteral reflux and neonates/infants with prenatal hydronephrosis. J Urol 184:1145-1151

57. Lee JH, Choi HS, Kim JK, Won HS, Kim KS, Moon DH et al (2008) Nonrefluxing neonatal hydronephrosis and the risk of urinary tract infection. J Urol 179(4):1524-1528

58. Braga LH, Farrokhyar F, D'Cruz J, Pemberton J, Lorenzo AJ (2015) Risk factors for febrile urinary tract infection in children with prenatal hydronephrosis: a prospective study. J Urol 193(5 Suppl):1766-1771

59. Braga LH, D'Cruz J, Rickard M, Jegatheeswaran K, Lorenzo AJ (2016) The fate of primary nonrefluxing megaureter: a prospective outcome analysis of the rate of urinary tract infections, surgical indications and time to resolution. J Urol 195(4 Pt 2):1300-1305

60. Easterbrook B, Capolicchio JP, Braga LH (2017) Antibiotic prophylaxis for prevention of urinary tract infections in prenatal hydronephrosis: an updated systematic review. Can Urol Assoc J 11(1-2Suppl1):S3-S11

61. Herndon ACD, Cain MP (2009) AUA Update Series Lesson 3; 28 prenatal diagnosis of urological disease

62. Davenport MT, Merguerian PA, Koyle M (2013) Antenatally diagnosed hydronephrosis: current postnatal management. Pediatr Surg Int 29(3):207-214

63. Gloor JM, Ramsey PS, Ogburn PL Jr, Danilenko-Dixon DR, DiMarco CS, Ramin KD (2002) The association of isolated mild fetal hydronephrosis with postnatal vesicoureteral reflux. J Matern Fetal Neonatal Med 12(3):196-200

64. Zerin JM, Ritchey ML, Chang AC (1993) Incidental vesicoureteral reflux in neonates with antenatally detected hydronephrosis and other renal abnormalities. Radiology 187:157-160

65. Hodson EM, Wheeler DM, Vimalchandra D, Smith GH, Craig JC (2007) Interventions for primary vesicoureteric reflux. Cochrane Database Syst Rev 18:CD001532

66. Madden-Fuentes RJ, McNamara ER, Nseyo U, Wiener JS, Routh JC, Ross SS (2014) Resolution rate of isolated low-grade hydronephrosis diagnosed within the first year of life. J Pediatr Urol 10(4):639-644

67. Sencan A, Carvas F, Hekimoglu IC, Caf N, Sencan A, Chow J et al (2014) Urinary tract infection and vesicoureteral reflux in children with mild antenatal hydronephrosis. J Pediatr Urol 10(6):1008-1013

68. Ismaili K, Avni FE, Hall M, Brussels Free University Perinatal Nephrology (BFUPN) Study Group (2002) Results of systematic voiding cystourethrography in infants with antenatally diagnosed renal pelvis dilation. $J$ Pediatr 141:21-24

69. Ward VL (2006) Patient dose reduction during voiding cystourethrography. Pediatr Radiol 36(Suppl 2):168-172

70. Riccabona M (2004) Assement and management of newborn hydronephrosis. World J Urol 22:73-78

71. Yerkes E, Adams M, Pope J, Brock J (1999) Does every patient with hydronephrosis need voiding cystourethrography? J Urol 162:1218-1220

72. Garin EH, Olavarria F, Garcia Nieto V, Valenciano B, Campos A, Young L (2006) Clinical significance of primary vesicoureteral reflux and urinary antibiotic prophylaxis after acute pyelonephritis: a multicenter, randomized, controlled study. Pediatrics 117:626-632

73. Alconcher LF, Tombesi MM (2012) Natural history of bilateral mild isolated antenatal hydronephrosis conservatively managed. Pediatr Nephrol 27:1119-1123
74. de Kort EH, Bambang Oetomo S, Zegers SH (2008) The long-term outcome of antenatal hydronephrosis up to 15 millimetres justifies a noninvasive postnatal follow-up. Acta Paediatr 97:708-713

75. Szymanski KM, Al-Said AN, Pippi Salle JL, Capolicchio JP (2012) Do infants with mild prenatal hydronephrosis benefit from screening for vesicoureteral reflux? J Urol 188(2):576-581

76. Braga LH, Mijovic H, Farrokhyar F, Pemberton J, DeMaria J, Lorenzo AJ (2013) Antibiotic prophylaxis for urinary tract infections in antenatal hydronephrosis. Pediatrics 131(1):e251-e261

77. Kose E, Yavascan O, Turan O, Kangin M, Bal A, Alparslan C et al (2013) The effect of circumcision on the frequency of urinary tract infection, growth and nutrition status in infants with antenatal hydronephrosis. Ren Fail 35:1365-1369

78. Jodal U (1987) The natural history of bacteriuria in childhood. Infect Dis Clin North Am 1(4):713-729

79. Signorelli M, Cerri V, Taddei F, Groli C, Bianchi UA (2005) Prenatal diagnosis and management of mild fetal pyelectasis: implications for neonatal outcome and follow-up. Eur J Obstet Gynecol Reprod Biol 118:154-159

80. Nepple KG, Arlen AM, Austin JC, Cooper CS (2011) The prognostic impact of an abnormal initial renal ultrasound on early reflux resolution. J Pediatr Urol 7:462-466

81. Moorthy I, Joshi N, Cook JV, Warren M (2003) Antenatal hydronephrosis: negative predictive value of normal postnatal ultrasound-a 5-year study. Clin Radiol 58:964-970

82. Matsui F, Shimada K, Matsumoto F, Takano S (2008) Late recurrence of symptomatic hydronephrosis in patients with prenatally detected hydronephrosis and spontaneous improvement. J Urol 180:322-325 (discussion 5)

83. Gatti JM, Broecker BH, Scherz HC, Perez-Brayfield MR, Kirsch AJ (2001) Antenatal hydronephrosis with postnatal resolution: how long are postnatal studies warranted? Urology 57:1178

84. Ulman I, Jayanthi V, Koff S (2000) The long-term followup of newborns with severe unilateral hydronephrosis initially treated nonoperatively. J Urol 164:1101-1105

85. Hafez AT, McLorie G, Bagli D, Khoury A (2002) Analysis of trends on serial ultrasound for high grade neonatal hydronephrosis. J Urol 168:1518-1521

86. Coelho GM, Bouzada MCF, Lemos GS, Pereira AK, Lima BP, Oliveira EA (2008) Risk factors for urinary tract infection in children with prenatal renal pelvic dilatation. J Urol 179(1):284-289

87. Finnell SM, Carroll AE, Downs SM, Subcommittee on Urinary Tract Infection (2011) Technical report-diagnosis and management of an initial UTI in febrile infants and young children. Pediatrics 128(3):e749-e770

88. Lebowitz RL, Olbing H, Parkkulainen KV, Smellie JM, Tamminen-Mobius TE (1985) International system of radiographic grading of vesicoureteric reflux. International Reflux Study in Children. Pediatr Radiol 15:105-109

89. EISheemy MS, Ghoneima W, Abdelwahhab M, Aboulela W, Daw K, Shouman AM et al (2017) The role of voiding cystourethrography in asymptomatic unilateral isolated ureteropelvic junction obstruction: a retrospective study. J Pediatr Urol 13(2):206.e1-206.e7

90. Gordon I, Piepsz A, Sixt R, Auspices of Paediatric Committee of European Association of Nuclear Medicine (2011) Guidelines for standard and diuretic renogram in children. Eur J Nucl Med Mol Imaging 38:1175-1188

91. Koff SA, Campbell K (1992) Nonoperative management of unilateral neonatal hydronephrosis. J Urol 148(2 Pt 2):525-531

92. Nasser FM, Shouman AM, ElSheemy MS, Lotfi MA, Aboulela W, El Ghoneimy $M$ et al (2017) Dismembered pyeloplasty in infants 6 months old or younger with and without external trans-anastomotic nephrostent: a prospective randomized study. Urology 101:38-44

93. Morsi HA, Mursi K, Abdelaziz AY, Elsheemy MS, Salah M, Eissa MA (2013) Renal pelvis reduction during dismembered pyeloplasty: is it necessary? J Pediatr Urol 9(3):303-306

94. Ward VL, Strauss KJ, Barnewolt CE, Zurakowski D, Venkatakrishnan V, Fahey $\mathrm{FH}$ et al (2008) Pediatric radiation exposure and effective dose reduction during voiding cystourethrography. Radiology 249:1002 
95. Koff SA (2000) Postnatal management of antenatal hydronephrosis using an observational approach. Urology 55(5):609-611

96. Conway JJ, Maizels M (1992) The "well tempered" diuretic renogram: a standard method to examine the asymptomatic neonate with hydronephrosis or hydroureteronephrosis. A report from combined meetings of The Society for Fetal Urology and members of The Pediatric Nuclear Medicine Council-The Society of Nuclear Medicine. J Nuc Med 33(11):2047-2051
97. Shulkin BL, Mandell GA, Cooper JA, Leonard JC, Majd M, Parisi MT, Sfakianakis GN, Balon HR, Donohoe KJ (2008) Procedure guideline for diuretic renography in children 3.0. J Nucl Med Technol 36(3):162-168

\section{Publisher's Note}

Springer Nature remains neutral with regard to jurisdictional claims in published maps and institutional affiliations.
Submit your manuscript to a SpringerOpen ${ }^{\circ}$ journal and benefit from:

- Convenient online submission

- Rigorous peer review

- Open access: articles freely available online

- High visibility within the field

- Retaining the copyright to your article

Submit your next manuscript at $\boldsymbol{\nabla}$ springeropen.com 